\title{
Projected Future Changes in The Equatorial Wave Spectrum in CMIP6
}

\author{
Hagar Bartana ( $\nabla$ haggarb@gmail.com ) \\ Hebrew University of Jerusalem Fredy and Nadine Herrmann Institute of Earth Sciences \\ https://orcid.org/0000-0002-4278-4590 \\ Chaim Garfinkel \\ The Hebrew University of Jerusalem \\ Ofer Shamir \\ NYU: New York University \\ Jian Rao \\ Nanjing University of Information Science and Technology
}

\section{Research Article}

Keywords: convectively coupled equatorial waves, CMIP, Kelvin waves, Madden Julian Oscillation

Posted Date: November 17th, 2021

DOI: https://doi.org/10.21203/rs.3.rs-1064219/v1

License: (c) (1) This work is licensed under a Creative Commons Attribution 4.0 International License. Read Full License 


\title{
1 Projected Future Changes in the Equatorial Wave Spectrum in CMIP6
}

\author{
Hagar Bartana • Chaim I. Garfinkel . \\ Ofer Shamir • Jian Rao
}

6 Received: date / Accepted: date

7 Abstract The simulation of the Madden-Julian Oscillation (MJO) and convectively coupled equatorial waves (CCEWs) is considered in 13 state-ofthe-art models from phase 6 of the Coupled Model Intercomparison Project (CMIP6). We use frequency-wavenumber power spectra of the models and observations for Outgoing Longwave Radiation (OLR) and zonal velocity at 250 $\mathrm{hPa}$ (U250), and consider the historical and end-of-century projections for the SSP245 and SSP585 scenarios. The models simulate a spectrum quantitatively resembling that observed, though systematic biases exist. MJO and Kelvin waves (KW) are mostly underestimated, while equatorial Rossby waves (ER) are overestimated. The models project a moderate future increase in power for the MJO, a robust increase for Kelvin waves (KW) and weaker power values for most other wavenumber-frequency combinations, including higher wavenumber ER. In addition to strengthening, KW also shift toward higher phase speeds (or equivalent depths). Models with a more realistic MJO in their control climate tend to simulate a stronger intensification, and models

Chaim I. Garfinkel

The Fredy and Nadine Herrmann Institute of Earth Sciences, Hebrew University of Jerusalem, Jerusalem, Israel

E-mail: chaim.garfinkel@mail.huji.ac.il

Ofer Shamir

Center for Atmosphere-Ocean Science, Courant Institute of Mathematical Sciences, New York, New York

Jian Rao

Key Laboratory of Meteorological Disaster, Ministry of Education (KLME)/Joint International Research Laboratory of Climate and Environment Change (ILCEC)/Collaborative Innovation Center on Forecast and Evaluation of Meteorological Disasters (CIC-FEMD), Nanjing University of Information Science and Technology, Nanjing, China

Hagar Bartana

The Fredy and Nadine Herrmann Institute of Earth Sciences, Hebrew University of Jerusalem, Jerusalem, Israel 
with a more realistic KW in their control climate tend to simulate a weaker intensification.

Keywords convectively coupled equatorial waves · CMIP · Kelvin waves · Madden Julian Oscillation

\section{Introduction}

The Madden-Julian Oscillation (MJO) is the dominant mode of intraseasonal (1-3 months) variability in the tropical atmosphere (Le et al, 2021; Ahn et al, 2017; Hung et al, 2013; Jiang et al, 2020; Madden and Julian, 1972; Zhang, 2005). It is characterized by eastward-propagating, planetary-scale envelopes of convective cloud clusters that are tightly coupled with the large-scale wind field. Its large spatial extent and low frequency (zonal wavenumbers 1-3 and 30-90 days period) distinguishes it from convectively coupled equatorial waves (CCEWs) and other disturbances (Le et al, 2021; Ahn et al, 2020, 2017; Hung et al, 2013; Jiang et al, 2020).

CCEWs are manifested as equatorially trapped, zonally propagating tropical circulations, and compose a nonnegligible fraction of submonthly tropical dynamical and convective variability. The CCEWs include Kelvin waves (KW), equatorial Rossby (ER), mixed Rossby-gravity (MRG), eastward inertio-gravity (EIG), and westward inertio-gravity (WIG) waves (Seo et al, 2012; Hung et al, 2013; Huang et al, 2013; Wheeler and Kiladis, 1999; Kiladis et al, 2009).

The MJO and CCEWs interact with a wide range of tropical weather and climate phenomena, including monsoonal systems, tropical cyclone activity, and the El Niño-Southern Oscillation (Le et al, 2021; Ahn et al, 2017; Hung et al, 2013). Furthermore, they also exhibit teleconnections to the extratropics, affecting regional hydroclimate, and influencing weather and climate phenomena in the mid-latitude and high-latitude regions (Yoo et al, 2012; Le et al, 2021; Ahn et al, 2020, 2017; Hung et al, 2013; Schwartz and Garfinkel, 2017; Raghavendra et al, 2019; Schwartz and Garfinkel, 2020). Therefore, they play an important role in the global climate system, and are a key source of predictability for the extended-range forecasts in both the tropics and extratropics (Hung et al, 2013; Raghavendra et al, 2019; Rao et al, 2021; Jiang et al, 2020; Zhang, 2005; Kiladis et al, 2009; Garfinkel and Schwartz, 2017; Vitart, 2017).

The ability of state-of-the-art coupled general circulation models (CGCMs) to accurately capture the MJO's magnitude, location, and dynamics is of vital importance for subseasonal-to-seasonal prediction (Le et al, 2021; Raghavendra et al, 2019; Jiang et al, 2020; Vitart, 2017). A wide range of factors, for example - air-sea coupling, vertical heating profile and cloud parameterization - have been shown to influence the strength of the MJO and CCEWs in models (Le et al, 2021; Ahn et al, 2020, 2017; Hung et al, 2013; Wang and Li, 2017; Raghavendra et al, 2019; Seo et al, 2012; Jiang et al, 2015; Huang et al, 2013; Jiang et al, 2020).

Despite the importance of the MJO, very few models from phase 5 of the Coupled Model Intercomparison Project (CMIP) were able to simulate a 
realistic MJO (Raghavendra et al, 2019). Although only a few studies have investigated the performance of CMIP6 models in capturing CCEWs and the MJO so far, there seems to be a robust improvement in the representation of the MJO in the CMIP6 models. Specifically, CMIP6 models with an improved representation of convection show significantly better results (Ahn et al, 2020). Nevertheless, they still tend to underestimate the variability contributed by the MJO (Le et al, 2021; Ahn et al, 2020), even as they exhibit reasonable spectral power or total variance within the intraseasonal timescales as compared to observations.

While considering the limitations of the models, they allow us a glimpse of future change possibilities. Previous work cautiously predicts an intensification of the MJO and KW. (Chang et al, 2015; Bui and Maloney, 2019; Raghavendra et al, 2019; Maloney et al, 2019). Furthermore, by the end of the century the MJO might have less influence on extratropical phenomena in some regions (Bui and Maloney, 2019; Chang et al, 2015), but a stronger influence in others (Samarasinghe et al, 2021).

In this study, we analyze simulations of the MJO and CCEWs in 13 CMIP6 models. We specifically focus on the KW and ER, as they are strongly associated with the MJO and have strong mutual influence.

Section 2 will describe the data and methods. The results (section 3) are divided into two subsections: analysis of historical biases, and an analysis of future assessments. Discussion and conclusions are presented in section 4 .

\section{Data and Methods}

\subsection{Data}

Thirteen CMIP6 models are analyzed in this study, chosen based on the availability of daily data for outgoing long wave radiation (OLR) and zonal velocity at $250 \mathrm{hPa}$ (U250) for both the historical scenario and the two future scenarios: SSP245 and SSP585. The SSP585 scenario includes an additional radiative forcing of $8.5 \mathrm{~W} / \mathrm{m}^{2}$ by the year 2100 while the SSP245 scenario includes an additional radiative forcing of $4.5 \mathrm{~W} / \mathrm{m}^{2}$ by the year 2100 (Meinshausen et al, 2020). The years used for the historical data for the models are 1850-2014, except for FGOALS-g3 which has data up to 2016. Output from the historical simulations are compared to U250 from ERA5 (Hersbach et al, 2020) and to the interpolated OLR product from NOAA (Liebmann and Smith, 1996). Years used for U250 are 1979-2020, and for OLR from June 1974 through May 2021. The OLR observational data has enhanced power around $(k, \omega)=(14,0.1)$ that is likely an artefact of the sampling of the polar-orbiting satellites (Wheeler and Kiladis, 1999). This area is not associated with either the MJO or any of the CCEWs so we ignore it. Years used for future assessments are 2015-2100, except for FGOALS-g3 which has data only until 2099. Table 1 summarizes the data products used. 


\begin{tabular}{|l|l|l|}
\multicolumn{2}{|c}{ Table 1: Data products used } \\
\hline \multirow{3}{*}{ obs } & data source & reference \\
\hline \multirow{5}{*}{ CMIP6 } & NOAA OLR & Liebmann and Smith (1996) \\
\cline { 2 - 3 } & ERA-5 & Hersbach et al (2020) \\
\cline { 2 - 3 } & BCC-CSM2-MR & Wu et al (2019) \\
\cline { 2 - 3 } & CESM2 & Danabasoglu et al (2020) \\
\cline { 2 - 3 } & CNRM-CM6-1 & Voldoire et al (2019) \\
\cline { 2 - 3 } & CNRM-ESM2-1 & Séférian et al (2019) \\
\cline { 2 - 3 } & EC-Earth3 & Döscher et al (2021) \\
\cline { 2 - 3 } & FGOALS-g3 & Li et al (2020) \\
\cline { 2 - 3 } & GFDL-CM4 & Dunne et al (2020) \\
\cline { 2 - 3 } & INM-CM4-8 & Volodin et al (2019) \\
\cline { 2 - 3 } & INM-CM5-0 & Vorobyeva and Volodin (2021) \\
\cline { 2 - 3 } & MIROC6 & Tatebe et al (2019) \\
\cline { 2 - 3 } & MPI-ESM1-2-HR & Müller et al (2018) \\
\cline { 2 - 3 } & MPI-ESM1-2-LR & Mauritsen et al (2019) \\
\cline { 2 - 3 } & UKESM1-0-LL & Sellar et al (2019) \\
\hline
\end{tabular}

Table 1 The data sources used in this study.

We focus on OLR and U250 for two reasons. OLR allows us to compare to previous work using previous CMIP generations and also to observations (Le et al, 2021; Raghavendra et al, 2019). We elect to focus on U250 for future projections because of its relationship with upper level divergence. Upper level divergence and divergent outflow leads to teleconnections in mid-latitudes (Sardeshmukh and Hoskins, 1988; Hoskins and Karoly, 1981), and hence an increase, say, in MJO activity of U250 may be expected to lead to stronger or more frequent teleconnections. Exploring this possibility is left for future work, and in particular we note the recent study of Jenney et al (2021) who find that changes in the subtropical mean state may be more important than changes in the MJO itself for future changes in MJO teleconnections.

\subsection{Methods}

We use the open-source wkSpaceTime routine of the NCAR Command Language, which implements the analysis described in Wheeler and Kiladis (1999) without the tropical depression filter used in Kiladis et al (2009). The results of the following sections were obtained using a temporal window of 96 days with an overlap of 10 days between consecutive windows, and a meridional window of $15^{\circ} S-15^{\circ} \mathrm{N}$. We overlay on the spectra the theoretical Matsuno wave-modes for equivalent depths of $10 \mathrm{~m}, 30 \mathrm{~m}$ and $90 \mathrm{~m}$, as differences between the $\beta$ plane solutions of Matsuno and the exact spherical solutions are small for the parameter regime of Earth's tropics (Garfinkel et al, 2017; Paldor et al, 2013; Paldor, 2015).

All data is processed and presented using a wavenumber-frequency $(\omega-\mathrm{k})$ power spectrum for the different parameters and scenarios for each model, for the multi-model mean (hereafter MMM) and for the observational data. All figures show the $\log _{10}$ of the spectrum (accordingly, all figures showing 


\section{Historical Symmetric OLR}
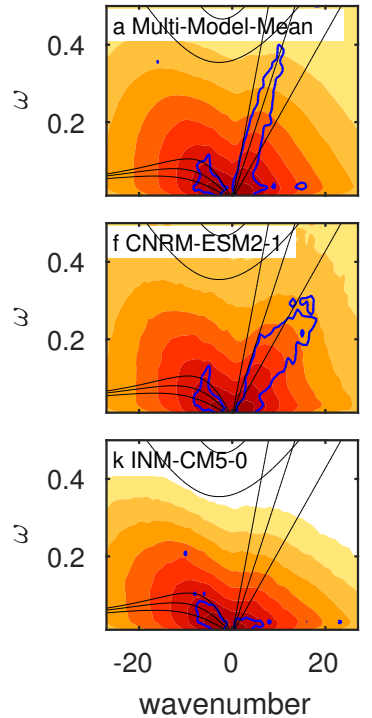
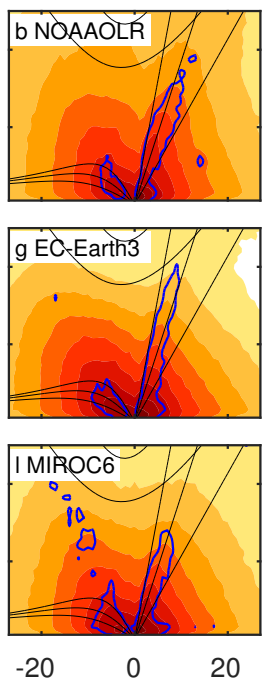

wavenumber
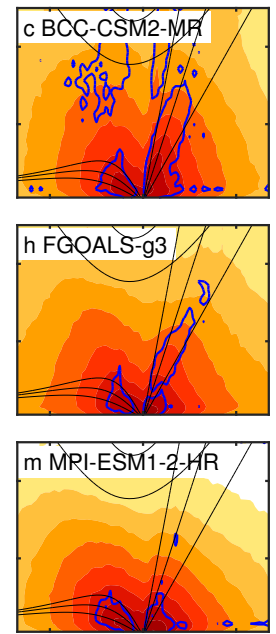

$-20 \quad 0 \quad 20$
wavenumber
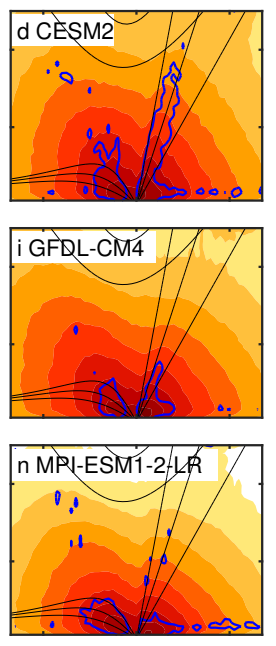

$-20$

wavenumber
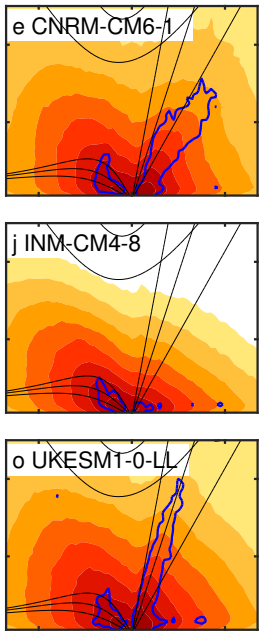

$-20$

0

20

wavenumber

Fig. $1 \log _{10}$ of the $\omega$-k power spectra of the symmetric component of raw OLR $\left(W / m^{2}\right)^{2} * s$ data for all models and observations: (a) is the MMM, (b) is OLR observations, and (c-o) are the models. Contour interval is 0.3. Black lines are the dispersion curves of equatorial waves for equivalent depths of $10 \mathrm{~m}, 30 \mathrm{~m}$ and $90 \mathrm{~m}$. A blue contour indicates where the power divided by the background equals 1.2.

differences between spectra correspond to the $\log _{10}$ of the ratio). For each spectrum, the total power is also calculated. After analyzing the historical biases and the relationship between biases in U250 and in OLR, we analyze the future projections. We focus on the symmetric component of the spectrum.

\section{Changes in Tropical Spectrum}

\subsection{Historical Bias}

Figure 1 shows the $\omega$-k power spectra of the symmetric component of OLR in the historical simulations. Blue contours indicate regions in which the power exceeds the background spectrum by at least $20 \%$, hence showing the power in the MJO and CCEWs that can be distinguished from the background turbulent red-noise (Garfinkel et al, 2021), and such a ratio is statistically significant at the $95 \%$ level even in the relatively short observational record (Shamir et al, 2021). Observations clearly show power exceeding the background spectrum for $\omega$-k combinations associated with the MJO, KW and ER, and so do many of the models and the MMM. The MMM shows a generally good representation of both the MJO, KW and ER as compared to observations, as represented by 


\section{Historical Symmetric OLR: model-observations}
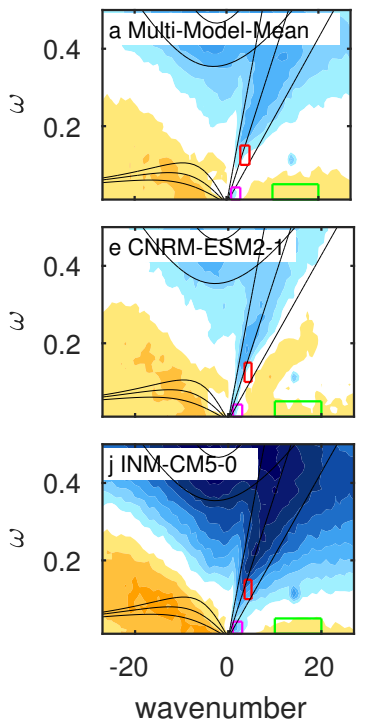
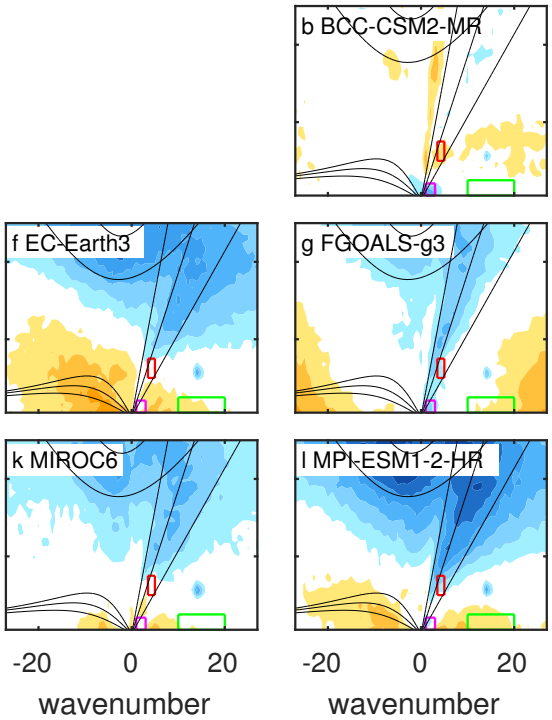
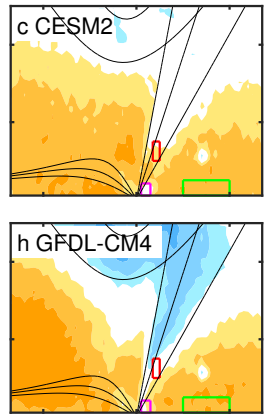

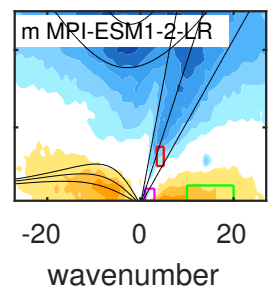

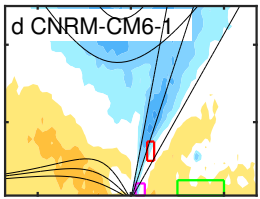
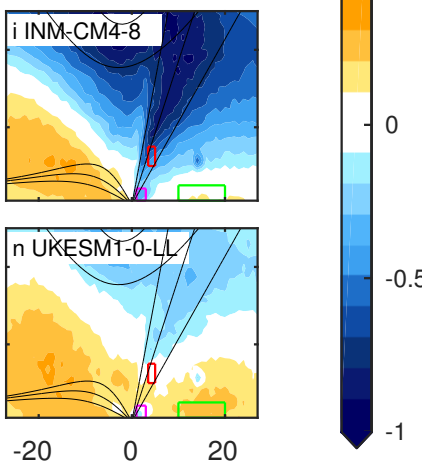

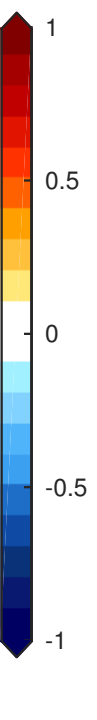

Fig. 2 Difference in the $\omega$-k power spectra (log-scaled) of the symmetric component of raw OLR $\left(W / m^{2}\right)^{2} * s$. (a) MMM, and (b-o) each of the models. Contour interval is 0.1. Black lines are the dispersion curves of equatorial waves for equivalent depths of $10 \mathrm{~m}, 30 \mathrm{~m}$ and 90m. Rectangles mark the areas for the correlation graphs (Figures 5, 7, 8): green marks areas without a theoretical dry wave $(10 \leq k \leq 20,20 \leq T \leq 96$ days), magenta marks $\omega$-k combinations in the vicinity of the Madden Julian Oscillation $(1 \leq k \leq 3,24 \leq T \leq 96$ days) and red marks $\omega$-k combinations in the vicinity of the Kelvin wave $(3.5 \leq k \leq 5$, $7 \leq T \leq 10$ days).

the blue contour. The KW is simulated in most models, though not all models capture a realistic phase speed: in some models the KW propagates too slowly (e.g. both CNRM and FGOALS-g3) while in others it propagates too fast (e.g. EC-Earth3).

The fidelity of the MMM and of each model is more easily visualized by computing the bias with respect to observations, and this bias is shown in Figure 2 (note that the bias is defined here as the difference between the $\log _{10}$ of the power spectra, i.e. the $\log _{10}$ of the ratio of modeled to observed power). BCC-CSM2-MR captures the spectrum most accurately. The rest of the models, as well as the MMM, generally overestimate low frequencies except for low wavenumbers, and underestimate higher frequencies. The magnitude of the bias differs between the models, and is particularly pronounced for the INM models (i.e., INM-CM4-8 and INM-CM5-0).

Similar to CMIP5, many models have a too-weak MJO bias, though in Figure 2 this bias is most notable in four models: BCC-CSM2-MR, FGOALS-g3, INM-CM4-8, INM-CM5-0. While the other models may simulate a reasonable amount of power for $\omega$-k values associated with the MJO, these other models 


\begin{tabular}{|c|c|c|c|c|c|}
\hline \multicolumn{6}{|c|}{ Total power } \\
\hline model no. & model name & OLR bias & u 250 bias & SSP245 difference & SSP585 difference \\
\hline $\mathrm{a}$ & BCC-CSM2-MR & 50.7 & 507.6 & -119.8 & -222.4 \\
\hline $\mathrm{b}$ & CESM2 & 346.3 & 224.4 & -77.9 & -146.3 \\
\hline $\mathrm{c}$ & CNRM-CM6-1 & -68.4 & 55.9 & -110.2 & -174.3 \\
\hline $\mathrm{d}$ & CNRM-ESM2-1 & -51.5 & 39.7 & -101.0 & -159.8 \\
\hline $\mathrm{e}$ & EC-Earth3 & -293.2 & -242.2 & 2.3 & -25.0 \\
\hline f & FGOALS-g3 & -99.5 & -167.9 & -107.2 & -186.6 \\
\hline g & GFDL-CM4 & 216.2 & -30.1 & -27.5 & -72.2 \\
\hline $\mathrm{h}$ & INM-CM4-8 & -927.0 & -770.8 & -60.7 & -96.0 \\
\hline i & INM-CM5-0 & -885.0 & -739.3 & -27.5 & -51.5 \\
\hline $\mathrm{j}$ & MIROC6 & -327.1 & 35.8 & -60.9 & -89.5 \\
\hline $\mathrm{k}$ & MPI-ESM1-2-HR & -532.2 & -8.2 & -70.8 & -108.9 \\
\hline 1 & MPI-ESM1-2-LR & -474.3 & 0.4 & -66.0 & -103.1 \\
\hline $\mathrm{m}$ & UKESM1-0-LL & -13.6 & 207.4 & -155.1 & -265.1 \\
\hline
\end{tabular}

Table 2 Summary of total power of each model for OLR and u 250 bias, and for differences between future assessments and bias (u 250).

simulate too much power at low frequencies at other wavenumbers however, and hence the MJO is not as important at accounting for intraseasonal variability in essentially all (BCC-CSM2-MR the lone exception) models as compared to observations.

Biases for KW are even more common. On Figure 1, only two-thirds of the models simulate enhanced power above the background spectrum at $\omega-\mathrm{k}$ combinations corresponding to the KW, with the INM, GFDL-CM4 and MPI models struggling most. On Figure 2, we compare to observations rather than each model's background spectrum. Six models are reasonable (BCC-CSM2MR, CESM2, EC-Earth3, GFDL-CM4, MIROC6 and UKESM1-0-LL), three slightly underestimate KW (CNRM-CM6-1, CNRM-ESM2-1, FGOALS-g3), and the remaining four (INM-CM4-8, INM-CM5-0, MPI-ESM1-2-HR, MPIESM1-2-LR) have biases exceeding a factor of three $\left(=10^{0.5}\right)$.

In contrast to the MJO and KW, all of the models capture enhanced power for ER compared to each model's background spectrum in Figure 1. When compared to observations (Figure 2), FGOALS-g3 and MIROC6 simulate a realistic amount of ER power, most of the models overestimate it and BCCCSM2-MR slightly underestimates it.

Table 2 shows the total power of the models compared to the observations (third column from the left). Most models have a negative total bias, except for BCC-CSM2-MR, CESM2, GFDL-CM4. UKESM1-0-LL has the lowest absolute value of total bias, though this is the net of too-strong low frequency and too-weak high frequency variability.

We now switch our focus to the U250hPa $\omega$-k power spectra (Figure 3), as our assessment of future changes are based on this variable. Compared to the OLR spectra, the symmetric component of historical U250 $\omega$-k power spectra (Figure 3 ) is more confined to lower values of both wavenumber and frequency. While the enhanced power in the vicinity of the KW is clear, MJO and ER are less evident (also if we lower the threshold for the blue contour on Figure 3, not shown). The power at negative low wavenumbers and frequency of approx- 


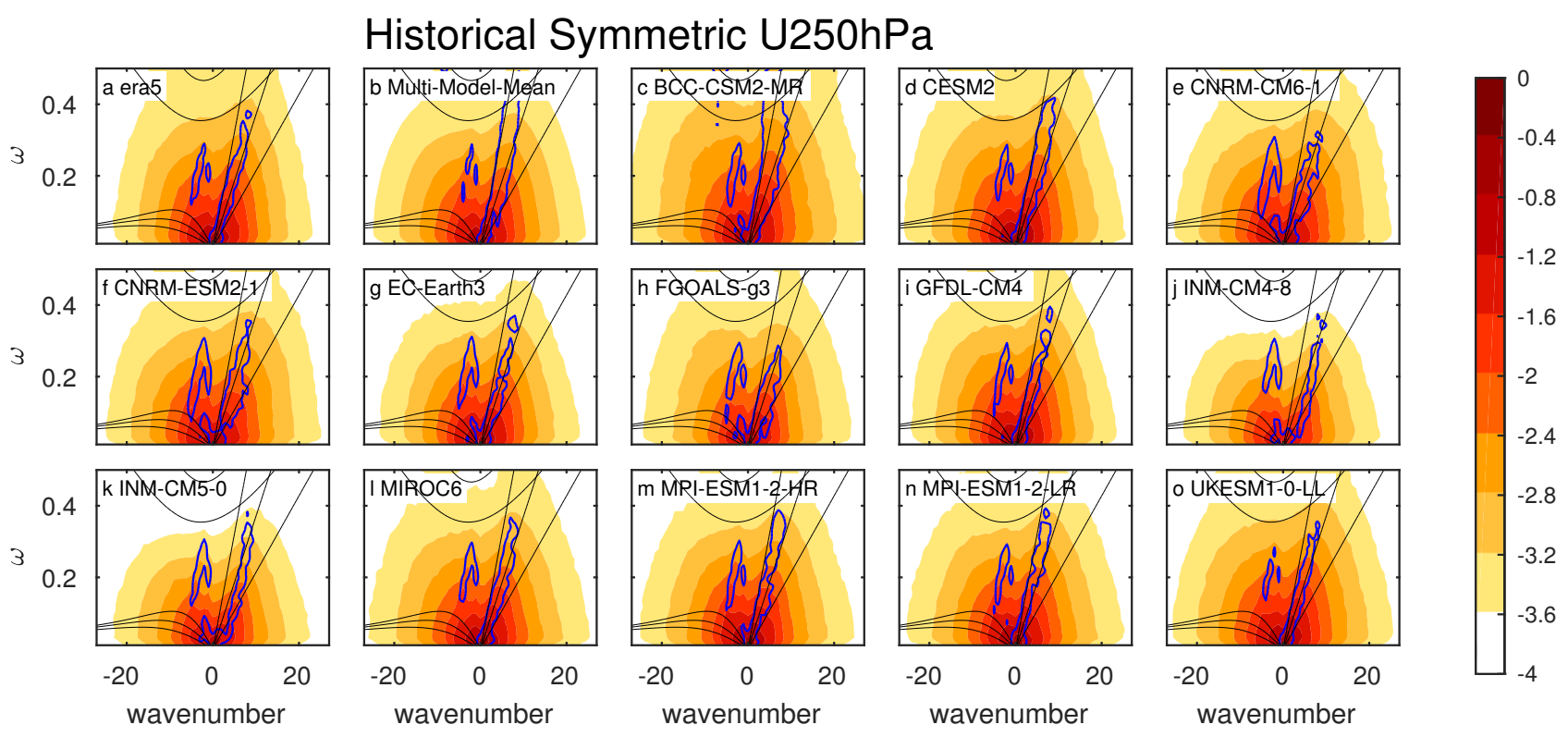

Fig. $3 \log _{10}$ of the $\omega$-k power spectra of the symmetric component of zonal wind $(\mathrm{m} / \mathrm{s})^{2} * s$ at $250 \mathrm{hPa}$ for all models and observations: (a) is observations, (b) is the MMM, and (c-o) are the models. Contour interval is 0.4. Black lines are the dispersion curves of equatorial waves for equivalent depths of $10 \mathrm{~m}, 30 \mathrm{~m}$ and $90 \mathrm{~m}$. A blue contour indicates where the power divided by the background equals 1.35 .

imately 0.1-0.3 corresponds to the external Rossby-Haurwitz waves (Hendon and Wheeler, 2008), which are not in the scope of this study. Looking at the MMM spectrum, there is a general overestimation of power at lower frequencies (including MJO and ER), and underestimation at higher frequencies. This bias towards low frequencies is similar to that for OLR.

Looking at the U250 bias of the models relative to observations (Figure 4), there is a systematic tendency for too little power at low wavenumbers relative to larger wavenumbers, and also relatively more power at low frequencies than at high frequencies. The net effect is that for most models (the exceptions are BCC-CSM2-MR, CESM2, FGOALS-g3, and GFDL-CM4) biases take the form of a triangle. Individual models also suffer from additional biases. BCCCSM2-MR, CESM2 and UKESM1-0-LL have a positive bias for most of the spectrum (BCC-CSM2-MR particularly biased), while the bias of EC-Earth3, FGOALS-g3, INM-CM4-8 and INM-CM5-0 is mostly negative (INM-CM4-8 and INM-CM5-0 bias values are particularly low and are an outlier). The sum of the biases for all values of $\omega$ and $k$ are lower however (see Table 2), because the total negative and positive biases within each spectrum compensate and cancel. Specifically, CNRM-CM6-1, CNRM-ESM2-1, MIROC6, MPI-ESM1-2HR and MPI-ESM1-2-LR have a somewhat similar distribution of negative and positive bias, which is also reflected in the MMM spectrum. The bias in 


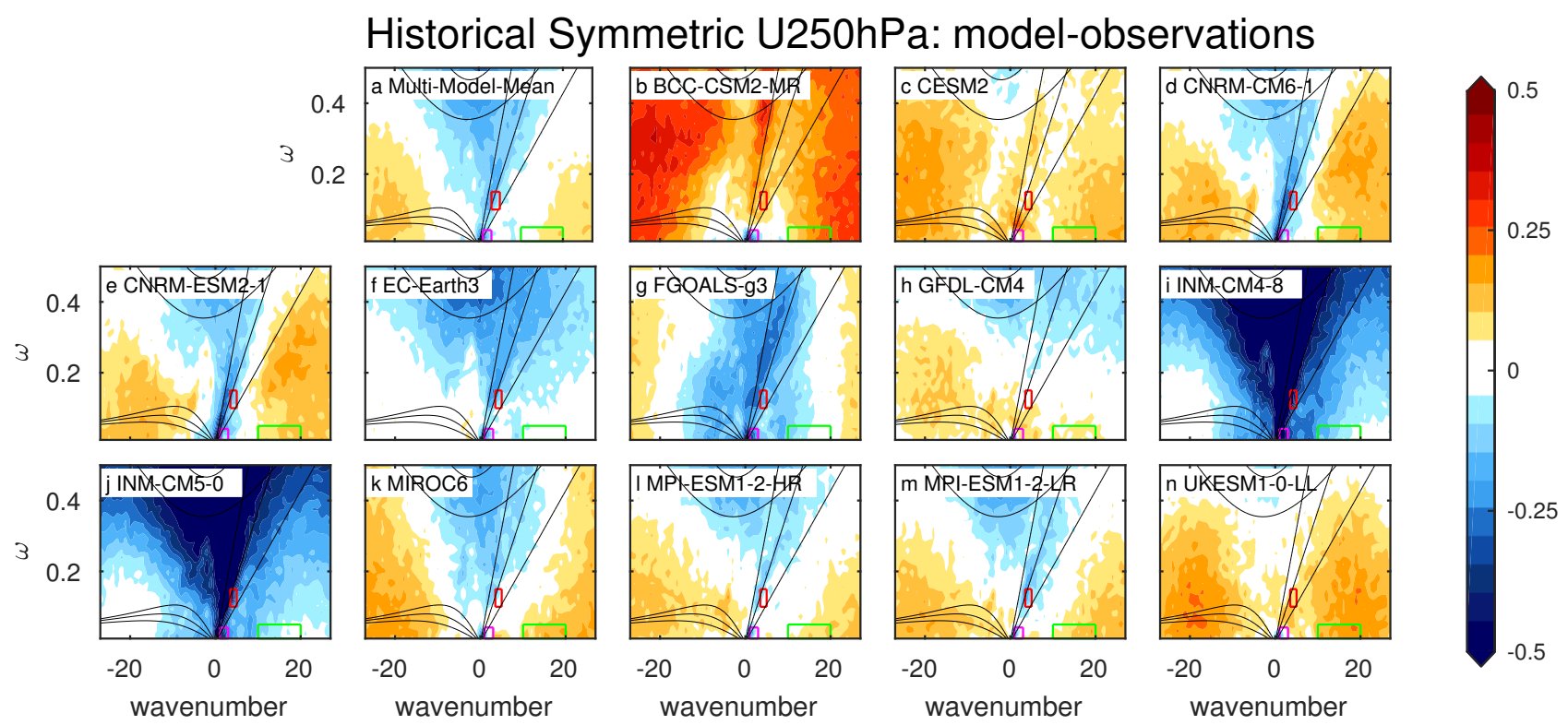

Fig. 4 Difference in the $\omega$-k power spectra (log-scaled) of the symmetric component of raw zonal velocity $(\mathrm{m} / \mathrm{s})^{2} * s$ at $250 \mathrm{hPa}$ : (a) MMM, and (b-o) each of the models. Contour interval is 0.05 . Black lines are the dispersion curves of equatorial waves for equivalent depths of $10 \mathrm{~m}, 30 \mathrm{~m}$ and $90 \mathrm{~m}$. Rectangles mark the areas for the correlation graphs (Figures $5,7,8)$ : green marks areas without a theoretical dry wave $(10 \leq k \leq 20,20 \leq T \leq 96$ days), magenta marks $\omega$-k combinations in the vicinity of the Madden Julian Oscillation $(1 \leq k \leq 3,24 \leq T \leq 96$ days) and red marks $\omega$-k combinations in the vicinity of the Kelvin wave $(3.5 \leq k \leq 5,7 \leq T \leq 10$ days $)$.

GFDL-CM4 differs from that in any other model, and it indicates too much westward propagation and not enough eastward propagation.

Most models have a too-weak MJO bias in U250, similar to the bias in OLR. Six models slightly underestimate it (BCC-CSM2-MR, CNRM-CM6-1, CNRM-ESM2-1, EC-Earth3, MPI-ESM1-2-HR, MPI-ESM1-2-LR) and three more (FGOALS-g3, INM-CM4-8, INM-CM5-0) underestimate it by more than a factor of two $\left(=10^{0.3}\right)$. Note that those three also underestimate the MJO in OLR.

In order to better quantify the relationship between biases in U250 and OLR, we compare the biases in Figure 5, using the areas within the colored rectangles on Figures 2 and 4. They represent regions of the $\omega$-k spectrum associated with specific phenomena: red represents $\mathrm{KW}$, magenta represents the MJO and green represents a region with no theoretical dry wave (i.e., the red background; Garfinkel et al (2021)). The phenomena are not confined into the boundaries of those areas, but the power values there are representative. Figure 5 shows the correlations between their OLR and U250 biases. The corresponding correlations between the raw data are similar (not shown). 


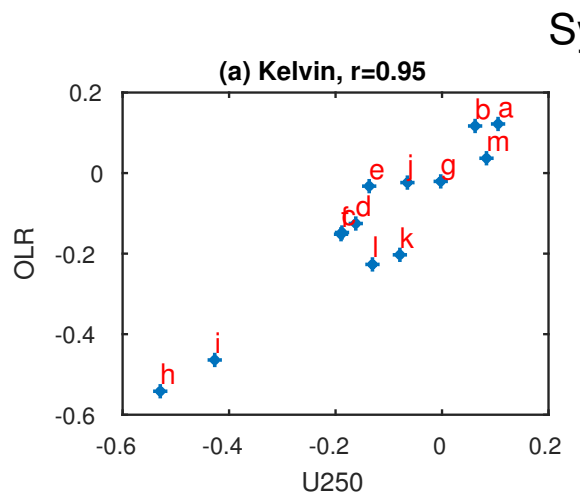

Symmetric U250 vs OLR

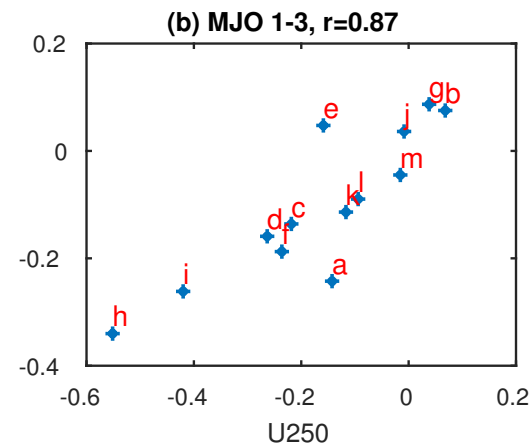

(c) no dry wave, $r=0.25$

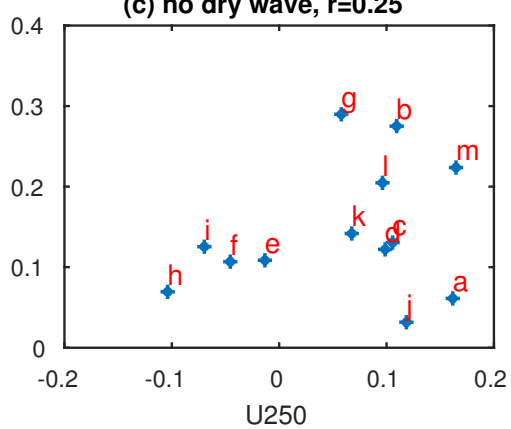

Fig. 5 correlations between biases in OLR and in zonal velocity at $250 \mathrm{hPa}$. $\omega$-k values: (a) KW: $3.5 \leq k \leq 5,7 \leq T \leq 10$ days; (b) MJO: $1 \leq k \leq 3,24 \leq T \leq 96$ days; (c) no dry wave: $10 \leq k \leq 20,20 \leq T \leq 96$ days. See the boxed regions on Figure 2. Letters correspond to the labeling of the models on Table 2).

Although the OLR and U250 spectra differ in the redness of the spectra in wavenumber (i.e., the slope in k), and models with biases in the background spectra of one variable do not necessarily have a bias in the background spectra of the other (Figure 5c), there is a tight relationship between models that struggle to represent the MJO or the KW in OLR vs. those that struggle to represent them in U250 (correlation exceeding 0.85). Out of the 13 models, two are noticeably poor: INM-CM4-8 and INM-CM5-0 (see the h and i points). They stand out in both OLR and U250 spectra, with their significantly low values for both MJO and KW, and also for the total bias (as shown in Table 2 ). The other models have relatively low bias for KW and MJO at U250 (less than $10^{0.2}$ or $58 \%$ ). Most models also do not have a significant bias for ER (not shown).

\subsection{Future Assessments}

Figure 6 presents the difference between the SSP585 future projection and the historical data for the symmetric component of the spectra. Although the models have an overall decrease in power, all models except for three (BCC-CSM2-MR, FGOALS-g3, MPI-ESM1-2-LR) project an intensification of KW. Most of them also project an increase in KW phase speed. One might expect future projections to be more reliable in models that are capable of more reasonably capturing the present climate, and hence Figure 7 considers the relationship between historical biases and future projections. Models with larger biases in their historical representation of the $\mathrm{KW}$ (i.e., the $\mathrm{KW}$ is too weak) tend to simulate larger future changes (CNRM, INM), while models with smaller biases tend to simulate weaker future changes (GFDL-CM4, MIROC6, MPI-ESM1-2-HR; Figure 7a). The correlation is only -0.47 , which 


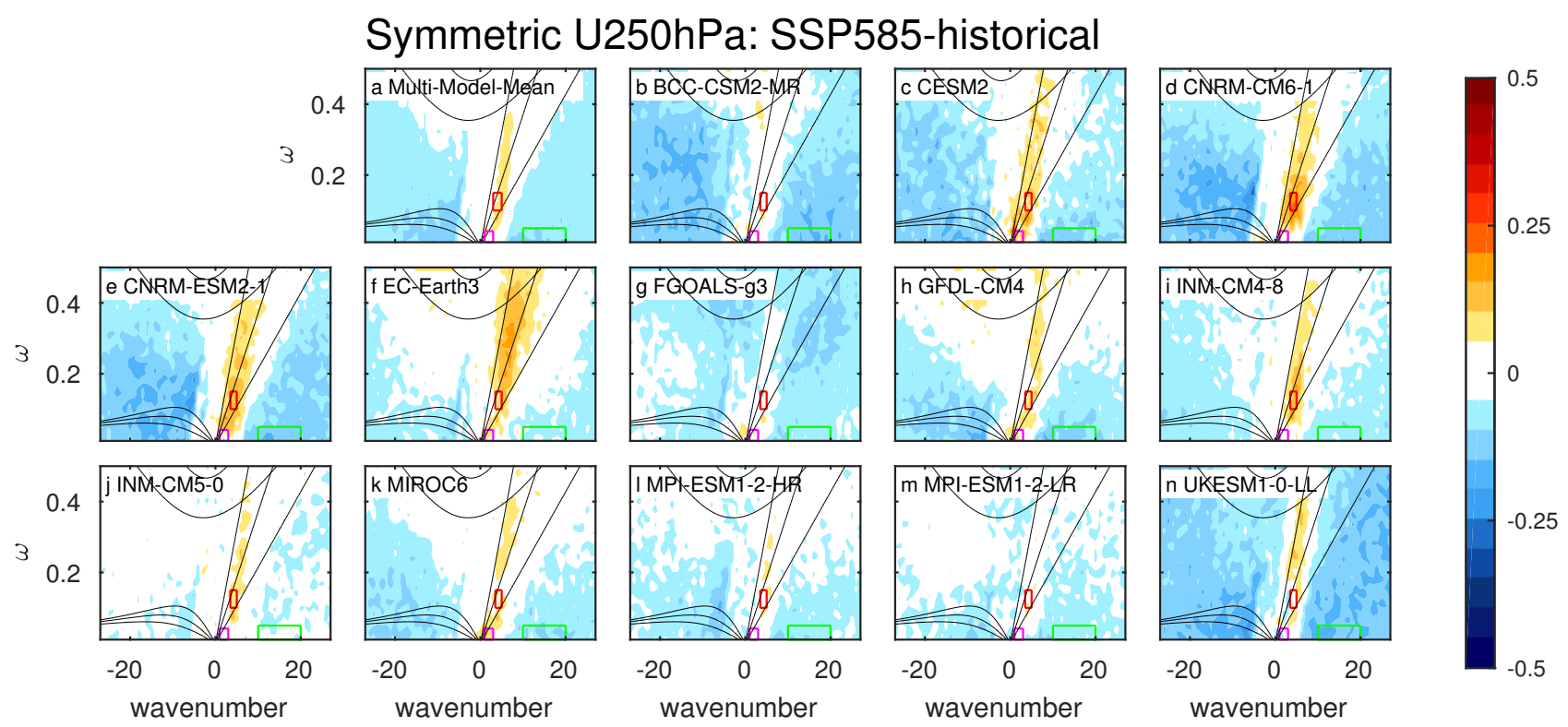

Fig. 6 Difference between the $\omega$-k power spectra (log-scaled) of the SSP585 projection and history for the symmetric component of raw zonal velocity $(\mathrm{m} / \mathrm{s})^{2} * s$ data at $250 \mathrm{hPa}$. (a) MMM, and (b-o) each of the models. Contour interval is 0.05. Black lines are the dispersion curves of equatorial waves for equivalent depths of $10 \mathrm{~m}, 30 \mathrm{~m}$ and $90 \mathrm{~m}$. Rectangles mark the areas for the correlation graphs (Figures 5, 7, 8): green marks areas without a theoretical dry wave $(10 \leq k \leq 20,20 \leq T \leq 96$ days), magenta marks $\omega$-k combinations in the vicinity of the Madden Julian Oscillation $(1 \leq k \leq 3,24 \leq T \leq 96$ days) and red marks $\omega$-k combinations in the vicinity of the Kelvin wave (3.5 $\leq k \leq 5,7 \leq T \leq 10$ days).

is not statistically significant at the $95 \%$ level assuming each model is an independent degree of freedom. Note, however, that the same relationship is also evident for the SSP245 projections (Figure 7d), and if the two projections are treated as independent samples then the overall correlations would be significant. Also, UKESM1-0-LL seems to have a weak future projection of KW, but Figure 6 shows that it is true only for the box we chose to focus on. In other $\omega$-k combinations associated with KW, UKESM1-0-LL projects an increase in KW.

Changes in the MJO are small in most models, though in the MMM there is a slight strengthening. Models with larger biases in their historical representation of the MJO (i.e. the MJO is too weak) tend to simulate little future change (CNRM, FGOALS-g3, INM), while models with smaller historical biases tend to simulate a future intensification of the MJO (CESM2, EC-Earth3, GFDL-CM4, MIROC6). However, the correlation is only 0.4 (Figure $7 \mathrm{~b}$ ), which is not statistically significant at the $95 \%$ level assuming each model is an independent degree of freedom, however a similar relationship is evident for the SSP245 projections (Figure 7e) which supports the robustness of this effect. CESM2, which overestimates the MJO in the historical 
Symmetric Historical vs Future
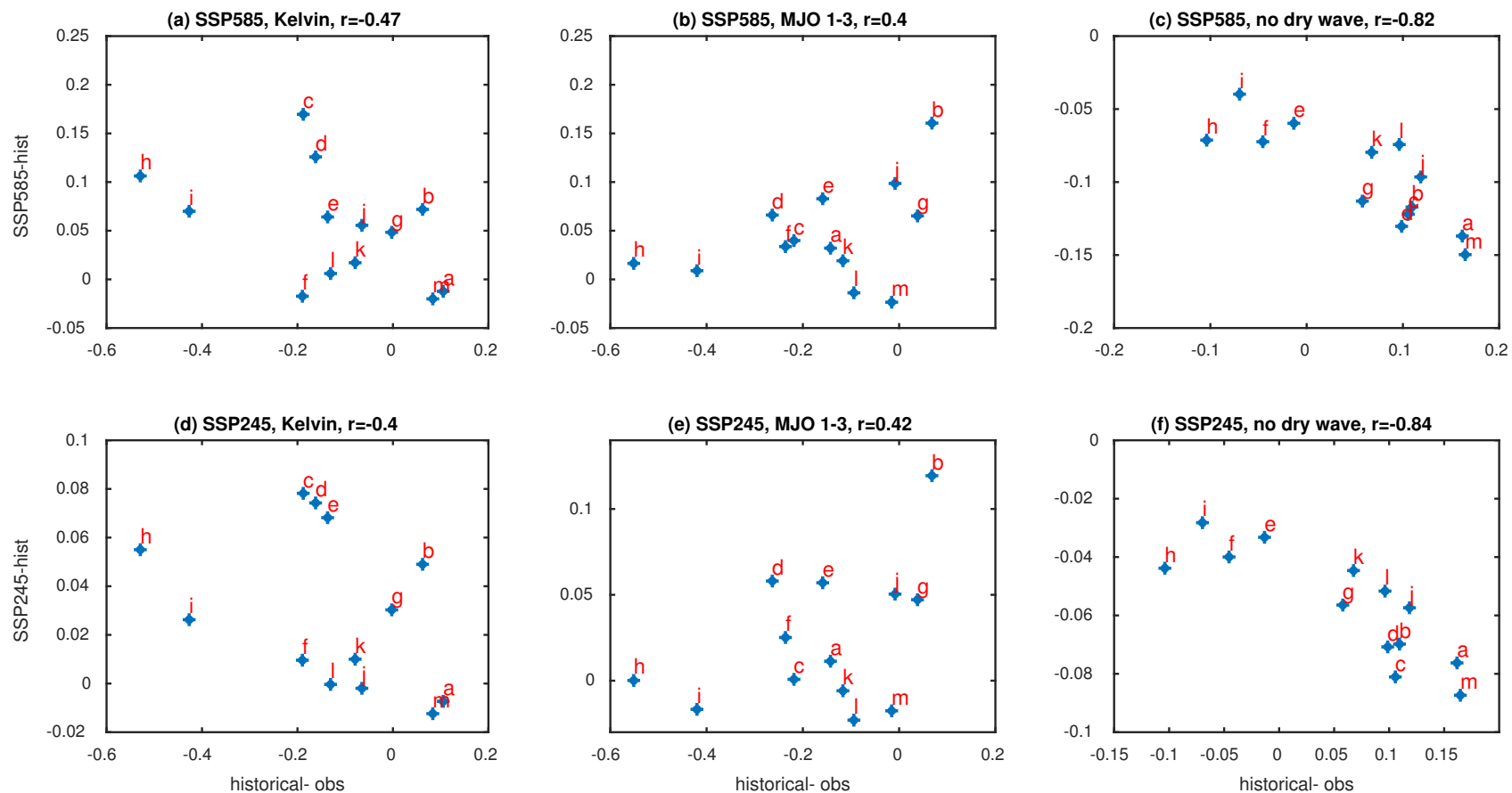

Fig. 7 Correlations between future forecasts and historical bias or the models:(a-c) SSP585 vs history, and (d-f) SSP245 vs history. $\omega$-k values: (a, d) KW: $3.5 \leq k \leq 5,7 \leq T \leq 10$ days; (b, e) MJO: $1 \leq k \leq 3,24 \leq T \leq 96$ days; (c, f) no dry wave: $10 \leq k \leq 20,20 \leq T \leq 96$ days. See the boxed regions on Figure 2 . Letters correspond to the labeling of the models on Table 2).

simulation the most, also shows the largest increase of power, while only MPIESM1-2-LR and UKESM1-0-LL (which have a negative bias) show a slight decrease. Changes in ER for wavenumbers less than 5 are not robust, however for larger wavenumbers ER activity is projected to decrease in all models.

For both SSP585 and SSP245 scenarios, all models show a decrease in total power (Table 2), except for one in SSP245 (EC-Earth3). They all project a decrease in power in the background, at least to some extent, and an increase of power in low wavenumbers (mostly eastward, but also westward). Models with too strong a background spectrum in their historical simulation robustly simulate a larger future decrease than models with a weak historical background spectrum (Figure $7 \mathrm{c}$ ). Five of them have a decrease exceeding $10^{0.15}$ (BCC-CSM2-MR, CESM2, CNRM-CM6-1, CNRM-ESM2-1, UKESM1-0-LL). FGOALS-g3 also has a total decrease similar to these five models (Table 2), however these five models all simulate a strengthening of $\mathrm{KW}$ so that the decrease in background is more pronounced. EC-Earth3 has the largest increase in $\mathrm{KW}$, and the smallest projected decrease in total power. 


\section{Symmetric SSP245 vs SSP585}
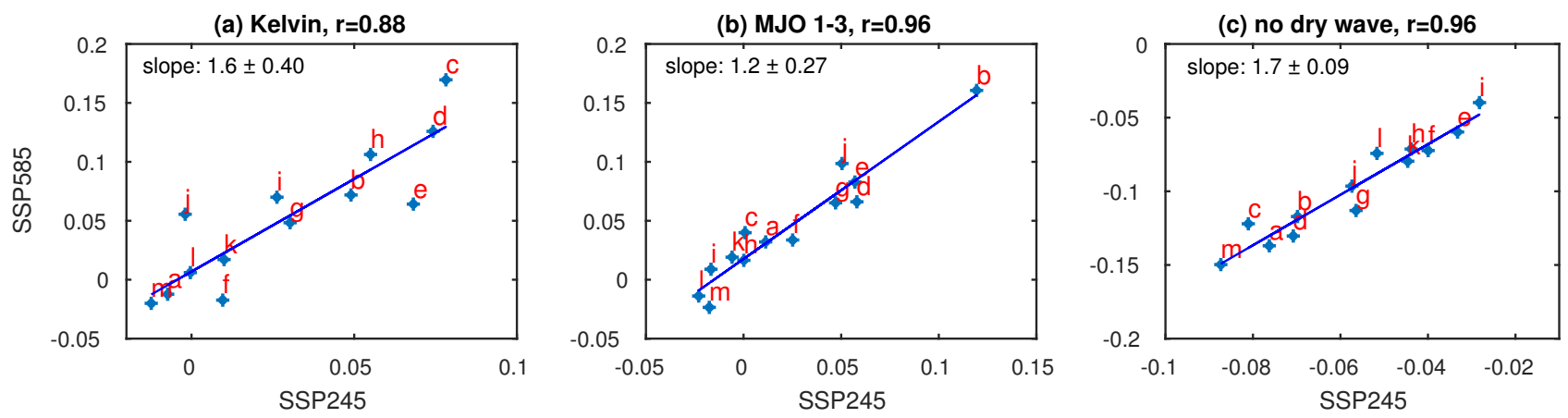

Fig. 8 correlations between SSP245 and SSP585.w-k values: (a) KW: $3.5 \leq k \leq 5,7 \leq$ $T \leq 10$ days; (b) MJO: $1 \leq k \leq 3,24 \leq T \leq 96$ days; (c) no dry wave: $10 \leq k \leq 20$, $20 \leq T \leq 96$ days. See the boxed regions on Figure 2 . Letters correspond to the labeling of the models on Table 2). A best-fit line and its slope, including 95\% confidence intervals, are shown for each panel.

Are the changes projected in SSP245 and in SSP585 linear? We consider this by contrasting projected changes in SSP245 vs. SSP585 in Figure 8 for each model. It is evident that there is a strong connection between the future projections, and a model with a stronger response for SSP245 also simulates a stronger response for SSP585. The correlation between future projections in SSP245 and in SSP585 is above 0.85 in all regions (Figure 8). For KW and background, the changes projected for SSP585 and SSP245 are proportional to the respective radiative forcings, with a slope of 1.7 for the background and 1.6 for KW. However, the projected change in the MJO is little different between the scenarios, with a slope of just 1.2. The reasons for these differences and similarities, along with the connection to the radiative forcings, should be a subject for future research.

\section{Summary and Discussion}

Assessing future change of the MJO and CCEWs is important both for their local tropical influence and their teleconnections to the extratropics. In this study we analyzed simulations of the MJO, KW and ER in 13 CMIP6 models, for U250 and OLR and three scenarios. While the spectra of U250 and OLR differ in the background spectrum, for the $\omega$-k combinations of the wavemodes, models' performance in e.g., U250 is robustly related to performance in OLR. For both U250 and OLR, most models underestimate the power associated with the MJO and KW, and overestimate the power associated with ER. The KW bias is most significant, and it is not always simulated at a realistic phase speed. On the other hand, ER biases are generally small. Out 
of the thirteen models, two (INM-CM4-8, INM-CM5-0) are noticeably poorer than the rest.

After establishing that most models perform well enough, we examined the future projections for SSP245 and SSP585 scenarios. We focused on the SSP585 scenario, which has more significant change, though results are generally similar for SSP245. Ten out of the thirteen models project a clear intensification of KW relative to their historical simulation. While this intensification is stronger in models with larger biases in their historical simulation, it is evident in many models with a relatively good historical climate as well. In addition to the intensification of $\mathrm{KW}$, the models project that $\mathrm{KW}$ phase speeds will also increase. In contrast, the background spectra for essentially all $\omega$-k values and for larger-wavenumbers ER is projected to weaken. Projected changes in the ER for small wavenumbers are less pronounced. Although INM-CM4-8 and INM-CM5-0 simulate the historical data poorly, their future projections of KW and MJO are not significantly different than the rest of the models.

The MJO strengthens slightly in the multi-model mean and crucially also in models which simulate a more realistic MJO in the historical climate. This projection is based on the power spectrum of zonal wind at $250 \mathrm{hPa}$, and appears to stand in contrast to other studies indicating a weak change in the zonal velocity of the MJO, especially compared to the significant projected increase in precipitation (Jiang et al, 2020; Chang et al, 2015; Maloney et al, 2019). Furthermore, the gap between the MJO and KW grows as the KW shifts to higher phase speeds, and the background spectrum in the vicinity of the MJO weakens. The net effect is a more organized tropical circulation on intraseasonal timescales that may affect other phenomena in, say, the extratropics. Nevertheless, this projected strengthening is not uniformly simulated by all models nor are changes in SSP245 vs. SSP 585 proportional to the underlying radiative forcing, and more detailed investigation is needed into how the structure of the MJO (e.g., amplitude, regions of growth/decay) will change. In addition, future work should investigate whether this relative strengthening of the power of the MJO may affect its influence on the extratropics and potentially lead to improved forecast abilities. 


\section{Statements and Declarations}

All authors contributed to the study conception and design. Material preparation and data were provided by Jian Rao and Ofer Shamir. Ofer Shamir began the data analysis, and Hagar Bartana performed most of the data analysis. Chaim Garfinkel oversaw the project. The first draft of the manuscript was written by Hagar Bartana and all authors commented on previous versions of the manuscript. All authors read and approved the final manuscript. C. I. G. and H. B. are supported by the ISF-NSFC joint research program (grant No.3259/19) and by the European Research Council starting grant under the European Union's Horizon 2020 research and innovation program (Grant Agreement 677756). The authors have no competing interests to declare that are relevant to the content of this article.

Acknowledgements Interpolated OLR data provided by the NOAA/OAR/ESRL PSL Boulder, Colorado, USA, from their Web site at https://psl.noaa.gov/data/gridded/ data.interp_OLR.html. CMIP6 data is available from the ESGF website at https://esgf-node . llnl.gov/projects/cmip6/. Correspondence and requests for data should be addressed to C.I.G. (email: chaim.garfinkel@mail.huji.ac.il).

\section{References}

Ahn MS, Kim D, Sperber KR, Kang IS, Maloney E, Waliser D, Hendon $\mathrm{H}$ (2017) Mjo simulation in cmip5 climate models: Mjo skill metrics and process-oriented diagnosis. Climate Dynamics 49(11):4023-4045

Ahn MS, Kim D, Kang D, Lee J, Sperber KR, Gleckler PJ, Jiang X, Ham YG, Kim H (2020) Mjo propagation across the maritime continent: Are cmip6 models better than cmip5 models? Geophysical Research Letters 47(11):e2020GL087,250

Bui HX, Maloney ED (2019) Transient response of mjo precipitation and circulation to greenhouse gas forcing. Geophysical Research Letters 46(22):13,546-13,555

Chang CWJ, Tseng WL, Hsu HH, Keenlyside N, Tsuang BJ (2015) The madden-julian oscillation in a warmer world. Geophysical research letters 42(14):6034-6042

Danabasoglu G, Lamarque JF, Bacmeister J, Bailey D, DuVivier A, Edwards J, Emmons L, Fasullo J, Garcia R, Gettelman A, et al (2020) The community earth system model version 2 (cesm2). Journal of Advances in Modeling Earth Systems 12(2)

Döscher R, Acosta M, Alessandri A, Anthoni P, Arneth A, Arsouze T, Bergmann T, Bernadello R, Bousetta S, Caron LP, et al (2021) The ecearth3 earth system model for the climate model intercomparison project 6 . Geoscientific Model Development Discussions pp 1-90

Dunne J, Horowitz L, Adcroft A, Ginoux P, Held I, John J, Krasting J, Malyshev S, Naik V, Paulot F, et al (2020) The gfdl earth system model version 4.1 (gfdl-esm 4.1): Overall coupled model description and sim- 
ulation characteristics. Journal of Advances in Modeling Earth Systems 12(11):e2019MS002,015

Garfinkel C, Schwartz C (2017) Mjo-related tropical convection anomalies lead to more accurate stratospheric vortex variability in subseasonal forecast models. Geophysical research letters 44(19):10-054

Garfinkel CI, Fouxon I, Shamir O, Paldor N (2017) Classification of eastward propagating waves on the spherical earth. Quarterly Journal of the Royal Meteorological Society 143(704):1554-1564

Garfinkel CI, Shamir O, Fouxon I, Paldor N (2021) Tropical background and wave spectra: Contribution of wave-wave interactions in a moderately nonlinear turbulent flow. Journal of the Atmospheric Sciences 78(6):1773-1789

Hendon HH, Wheeler MC (2008) Some space-time spectral analyses of tropical convection and planetary-scale waves. Journal of the atmospheric sciences 65(9):2936-2948

Hersbach H, Bell B, Berrisford P, Hirahara S, Horányi A, Muñoz-Sabater J, Nicolas J, Peubey C, Radu R, Schepers D, Simmons A, Soci C, Abdalla S, Abellan X, Balsamo G, Bechtold P, Biavati G, Bidlot J, Bonavita M, De Chiara G, Dahlgren P, Dee D, Diamantakis M, Dragani R, Flemming J, Forbes R, Fuentes M, Geer A, Haimberger L, Healy S, Hogan RJ, Hólm E, Janisková M, Keeley S, Laloyaux P, Lopez P, Lupu C, Radnoti G, de Rosnay P, Rozum I, Vamborg F, Villaume S, Thépaut JN (2020) The era5 global reanalysis. Quarterly Journal of the Royal Meteorological Society n/a(n/a), DOI 10.1002/qj.3803, URL https://rmets .onlinelibrary . wiley.com/doi/abs/10.1002/qj.3803, https://rmets.onlinelibrary. wiley.com/doi/pdf/10.1002/qj. 3803

Hoskins BJ, Karoly DJ (1981) The steady linear response of a spherical atmosphere to thermal and orographic forcing. Journal of Atmospheric Sciences 38(6):1179-1196

Huang P, Chou C, Huang R (2013) The activity of convectively coupled equatorial waves in cmip3 global climate models. Theoretical and applied climatology 112(3):697-711

Hung MP, Lin JL, Wang W, Kim D, Shinoda T, Weaver SJ (2013) Mjo and convectively coupled equatorial waves simulated by cmip 5 climate models. Journal of Climate 26(17):6185-6214

Jenney AM, Randall DA, Barnes EA (2021) Drivers of uncertainty in future projections of madden-julian oscillation teleconnections. Weather and Climate Dynamics 2(3):653-673

Jiang X, Waliser DE, Xavier PK, Petch J, Klingaman NP, Woolnough SJ, Guan B, Bellon G, Crueger T, DeMott C, et al (2015) Vertical structure and physical processes of the madden-julian oscillation: Exploring key model physics in climate simulations. Journal of Geophysical Research: Atmospheres 120(10):4718-4748

Jiang X, Adames ÁF, Kim D, Maloney ED, Lin H, Kim H, Zhang C, DeMott CA, Klingaman NP (2020) Fifty years of research on the madden-julian oscillation: Recent progress, challenges, and perspectives. Journal of Geophysical Research: Atmospheres 125(17):e2019JD030,911 
Kiladis GN, Wheeler MC, Haertel PT, Straub KH, Roundy PE (2009) Convectively coupled equatorial waves. Reviews of Geophysics $47(2)$

Le PV, Guilloteau C, Mamalakis A, Foufoula-Georgiou E (2021) Underestimated mjo variability in cmip6 models. Geophysical Research Letters 48(12):e2020GL092,244

Li L, Yu Y, Tang Y, Lin P, Xie J, Song M, Dong L, Zhou T, Liu L, Wang L, et al (2020) The flexible global ocean-atmosphere-land system model gridpoint version 3 (fgoals-g3): description and evaluation. Journal of Advances in Modeling Earth Systems 12(9):e2019MS002,012

Liebmann B, Smith CA (1996) Description of a complete (interpolated) outgoing longwave radiation dataset. Bulletin of the American Meteorological Society $77(6): 1275-1277$

Madden RA, Julian PR (1972) Description of global-scale circulation cells in the tropics with a 40-50 day period. Journal of Atmospheric Sciences 29(6):1109-1123

Maloney ED, Adames ÁF, Bui HX (2019) Madden-julian oscillation changes under anthropogenic warming. Nature Climate Change 9(1):26-33

Mauritsen T, Bader J, Becker T, Behrens J, Bittner M, Brokopf R, Brovkin V, Claussen M, Crueger T, Esch M, et al (2019) Developments in the mpi-m earth system model version 1.2 (mpi-esm1. 2) and its response to increasing co2. Journal of Advances in Modeling Earth Systems 11(4):998-1038

Meinshausen M, Nicholls ZR, Lewis J, Gidden MJ, Vogel E, Freund M, Beyerle U, Gessner C, Nauels A, Bauer N, et al (2020) The shared socio-economic pathway (ssp) greenhouse gas concentrations and their extensions to 2500 . Geoscientific Model Development 13(8):3571-3605

Müller WA, Jungclaus JH, Mauritsen T, Baehr J, Bittner M, Budich R, Bunzel F, Esch M, Ghosh R, Haak H, et al (2018) A higher-resolution version of the max planck institute earth system model (mpi-esm1. 2-hr). Journal of Advances in Modeling Earth Systems 10(7):1383-1413

Paldor N (2015) Shallow water waves on the rotating Earth. Springer

Paldor N, De-Leon Y, Shamir O (2013) Planetary (rossby) waves and inertiagravity (poincaré) waves in a barotropic ocean over a sphere. Journal of Fluid Mechanics 726:123-136

Raghavendra A, Roundy PE, Zhou L (2019) Trends in tropical wave activity from the 1980s to 2016. Journal of Climate 32(5):1661-1676

Rao J, Garfinkel CI, Wu T, Lu Y, Lu Q, Liang Z (2021) The january 2021 sudden stratospheric warming and its prediction in subseasonal to seasonal models. Journal of Geophysical Research: Atmospheres p e2021JD035057

Samarasinghe SM, Connolly C, Barnes EA, Ebert-Uphoff I, Sun L (2021) Strengthened causal connections between the mjo and the north atlantic with climate warming. Geophysical Research Letters 48(5):e2020GL091,168

Sardeshmukh PD, Hoskins BJ (1988) The generation of global rotational flow by steady idealized tropical divergence. Journal of the Atmospheric Sciences 45(7):1228-1251

Schwartz C, Garfinkel CI (2017) Relative roles of the mjo and stratospheric variability in north atlantic and european winter climate. Journal of Geo- 
physical Research: Atmospheres 122(8):4184-4201

Schwartz C, Garfinkel CI (2020) Troposphere-stratosphere coupling in subseasonal-to-seasonal models and its importance for a realistic extratropical response to the madden-julian oscillation. Journal of Geophysical Research: Atmospheres 125(10):e2019JD032,043

Séférian R, Nabat P, Michou M, Saint-Martin D, Voldoire A, Colin J, Decharme B, Delire C, Berthet S, Chevallier M, et al (2019) Evaluation of cnrm earth system model, cnrm-esm2-1: Role of earth system processes in present-day and future climate. Journal of Advances in Modeling Earth Systems 11(12):4182-4227

Sellar AA, Jones CG, Mulcahy JP, Tang Y, Yool A, Wiltshire A, O'Connor FM, Stringer M, Hill R, Palmieri J, Woodward S, de Mora L, Kuhlbrodt T, Rumbold ST, Kelley DI, Ellis R, Johnson CE, Walton J, Abraham NL, Andrews MB, Andrews T, Archibald AT, Berthou S, Burke E, Blockley E, Carslaw K, Dalvi M, Edwards J, Folberth GA, Gedney N, Griffiths PT, Harper AB, Hendry MA, Hewitt AJ, Johnson B, Jones A, Jones CD, Keeble J, Liddicoat S, Morgenstern O, Parker RJ, Predoi V, Robertson E, Siahaan A, Smith RS, Swaminathan R, Woodhouse MT, Zeng G, Zerroukat M (2019) Ukesm1: Description and evaluation of the u.k. earth system model. Journal of Advances in Modeling Earth Systems 11(12):4513-4558, DOI 10.1029/ 2019MS001739

Seo KH, Choi JH, Han SD (2012) Factors for the simulation of convectively coupled kelvin waves. Journal of climate 25(10):3495-3514

Shamir O, Schwartz C, Garfinkel CI, Paldor N (2021) The power distribution between symmetric and antisymmetric components of the tropical wavenumber-frequency spectrum. Journal of the Atmospheric Sciences 78(6):1983-1998

Tatebe H, Ogura T, Nitta T, Komuro Y, Ogochi K, Takemura T, Sudo K, Sekiguchi M, Abe M, Saito F, et al (2019) Description and basic evaluation of simulated mean state, internal variability, and climate sensitivity in miroc6. Geoscientific Model Development 12(7):2727-2765

Vitart F (2017) Maddenjulian oscillation prediction and teleconnections in the s2s database. Quarterly Journal of the Royal Meteorological Society 143(706):2210-2220

Voldoire A, Saint-Martin D, Sénési S, Decharme B, Alias A, Chevallier M, Colin J, Guérémy JF, Michou M, Moine MP, et al (2019) Evaluation of cmip6 deck experiments with cnrm-cm6-1. Journal of Advances in Modeling Earth Systems 11(7):2177-2213

Volodin E, Mortikov E, Gritsun A, et al (2019) Inm inm-cm4-8 model output prepared for cmip6 cmip picontrol. version 20191230. Earth System Grid Federation https://doi org/1022033/ESGF/CMIP6 5080

Vorobyeva V, Volodin E (2021) Evaluation of the inm ras climate model skill in climate indices and stratospheric anomalies on seasonal timescale. Tellus A: Dynamic Meteorology and Oceanography 73(1):1-12

Wang L, Li T (2017) Convectively coupled kelvin waves in cmip5 coupled climate models. Climate Dynamics 48(3-4):767-781 
Wheeler M, Kiladis GN (1999) Convectively coupled equatorial waves: Analysis of clouds and temperature in the wavenumber-frequency domain. Journal of Atmospheric Sciences 56(3):374-399

${ }_{529} \mathrm{Wu} \mathrm{T}$, Lu Y, Fang Y, Xin X, Li L, Li W, Jie W, Zhang J, Liu Y, Zhang ${ }_{530}$ L, et al (2019) The beijing climate center climate system model (bcc-csm): The main progress from cmip5 to cmip6. Geoscientific Model Development 12(4):1573-1600

53 Yoo C, Lee S, Feldstein SB (2012) Mechanisms of arctic surface air temperature

534 change in response to the madden-julian oscillation. Journal of Climate

$535 \quad 25(17): 5777-5790$

${ }_{536}$ Zhang C (2005) Madden-julian oscillation. Reviews of Geophysics 43(2) 